\title{
Comparative chemistry of diffuse clouds
}

\section{II. $\mathrm{CN}, \mathrm{HCN}, \mathrm{HNC}, \mathrm{CH}_{3} \mathrm{CN} \& \mathrm{~N}_{2} \mathrm{H}^{+}$}

\author{
H. Liszt ${ }^{1}$ and R. Lucas ${ }^{2}$ \\ 1 National Radio Astronomy Observatory, 520 Edgemont Road, Charlottesville, VA 22903-2475, USA \\ 2 Institut de Radioastronomie Millimétrique, 300 rue de la Piscine, 38406 Saint Martin d'Hères, France
}

Received 18 October 2000 / Accepted 26 January 2001

\begin{abstract}
Using the Plateau de Bure interferometer, we observed the $\lambda 3 \mathrm{~mm}$ absorption lines of $\mathrm{CN}$, HCN and $\mathrm{HNC}$ from some of the diffuse clouds which lie toward our well-studied sample of compact extragalactic mm-wave continuum sources. The column densities of these species all vary by a factor of about fifty and are prominent in only a limited subset of the clouds seen in the most ubiquitous species such as $\mathrm{OH}, \mathrm{HCO}^{+}, \mathrm{C}_{2} \mathrm{H}$ and $\mathrm{C}_{3} \mathrm{H}_{2}$. We searched unsuccessfully for $\mathrm{CH}_{3} \mathrm{CN}$ and $\mathrm{N}_{2} \mathrm{H}^{+}$, which are underabundant compared to dark clouds, by factors of at least 10 and 100, respectively. The CN-HCN-HNC column densities vary strongly and non-linearly with $N\left(\mathrm{HCO}^{+}\right)$, for example, which probably best represents their variation with $\mathrm{H}_{2}$ as well. But their abundances are very tightly and linearly coupled to each other, varying in fixed proportion, as is the case for $\mathrm{OH}$ and $\mathrm{HCO}^{+}$, and (only slightly more loosely) for $\mathrm{C}_{2} \mathrm{H}$ and $\mathrm{C}_{3} \mathrm{H}_{2}$. Having measured one, it is hardly necessary to observe the two others in this group. We find $\langle N(\mathrm{HNC}) / N(\mathrm{HCN})\rangle=0.21 \pm 0.05,\langle N(\mathrm{CN}) / N(\mathrm{HCN})\rangle=6.8 \pm 1$. Such a small $N(\mathrm{HNC}) / N(\mathrm{HCN})$ ratio is typical of warmer gas in darker, denser environments, and is consistent with the notion of molecular formation in warmer media. The 6.8:1:0.21 ratio in diffuse gas is very different from TMC1 (6.8:4.5:4.5) where HCN and HNC are relatively much more abundant. It seems likely that the sequence of features with increasing column densities of the $\mathrm{CN}-\mathrm{HCN}$ family or $\mathrm{C}_{n} \mathrm{H}_{m}$-family molecules in diffuse gas actually represents a series of gas parcels of increasingly higher $\mathrm{H}_{2}$-fraction, number density, and molecular abundances, occurring over a relatively narrow interval of total hydrogen column density. Our experiment seems to have caught many molecules in the act of turning on - and turning each other on - in the diffuse interstellar medium.
\end{abstract}

Key words. ISM: molecules; abundances; clouds - ISM: structure Radio lines: ISM

\section{Introduction}

The presence of interstellar CN was shown by McKellar (1940) who identified the $R(0)$ line at $\lambda 387.4 \mathrm{~nm}$ (see also Swings \& Rosenfeld 1937; Adams 1941). The $R(1)$ line being undetectable, and taking as an upper limit on its equivalent width a value one-third that of $R(0)$, he calculated the "effective" or "rotational" temperature of interstellar space to be no more than $2.7 \mathrm{~K}$. McKellar qualified his conclusion, inquiring "if, indeed, the concept of such a temperature in a region with so low a density of both matter and radiation has any meaning". Of course it does have meaning, and $\mathrm{CN}$ has long been used to derive the temperature of the cosmic microwave background at a wavelength of $2.7 \mathrm{~mm}$, the contribution to the rotational excitation from collisions often being negligibly small (Thaddeus 1972; Crane et al. 1989; Black \& van Dishoeck 1991). Both $\mathrm{CH}$ and $\mathrm{CH}^{+}$, the other early molecules, are too light for their rotational structure to have been used in this way.

Send offprint requests to: H. S. Liszt, e-mail: hliszt@nrao.edu
Sixty years after the discovery of the CN lines we are still finding out what their presence in diffuse clouds actually means. Although it has not generally been regarded as much of a challenge to come up with rather conventional chemical schemes which account for the observed abundances of CN (Federman et al. 1994; Van Dishoeck \& Black 1986), these models beg larger questions which are the subject of this series of papers. Earlier we showed that the abundances of the closely related species $\mathrm{HCN}$ and HNC are much larger than expected in diffuse clouds seen toward compact extragalactic mm-wave continuum sources at galactic latitudes of $2-15^{\circ}$ (Lucas \& Liszt 1993, 1994). Given that the proximate source of $\mathrm{CN}$ in conventional models is the breakup of HCN and/or HNC and their immediate antecedents, it follows that our understanding of CN is at best accidental (see also Crawford \& Williams 1997).

In the first paper in this new series (Lucas \& Liszt 2000), we discussed the comparative chemistry of species in the $\mathrm{C}_{n} \mathrm{H}_{m}$-family of hydrocarbons whose most 


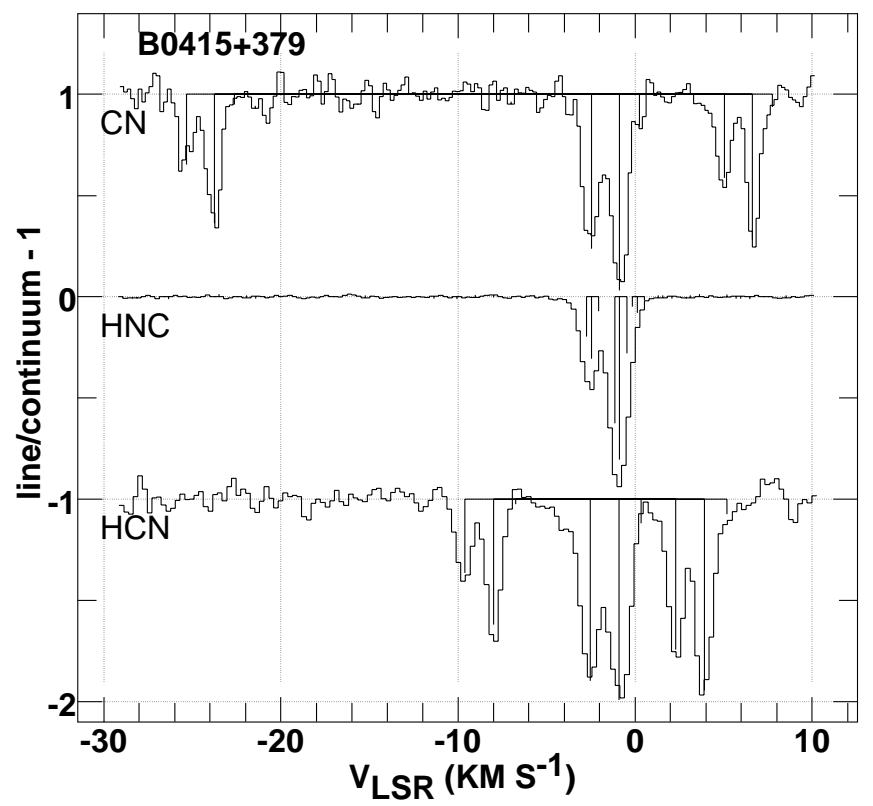

Fig. 1. CN, HCN and HNC absorption spectra toward B0415+379 (3C 111) showing the basic hyperfine structure in a fit involving three kinematic components (one of which is rather weak) for each species

abundant known members are $\mathrm{CH}, \mathrm{C}_{2} \mathrm{H}$ and $\mathrm{C}_{3} \mathrm{H}_{2}$. Here, we present results for the cyanogen- or CN-bearing molecules $\mathrm{CN}, \mathrm{HCN}$ and $\mathrm{HNC}$, based on a modest survey of $\lambda 3 \mathrm{~mm}$ absorption from clouds occulting a subset of our wider sample of compact extragalactic continuum sources (Lucas \& Liszt 1996). We find a remarkably tight linear correlation among all three, such that it is hardly necessary to observe the other two once the abundance of any one has been found: a similarly tight linear relation exists between $\mathrm{OH}$ and $\mathrm{HCO}^{+}$(Liszt \& Lucas 1994; Lucas \& Liszt 1996; Liszt \& Lucas 2000) and a somewhat looser but still quite good one exists between $\mathrm{C}_{2} \mathrm{H}$ and $\mathrm{C}_{3} \mathrm{H}_{2}$. By contrast, intercomparisons among the different families ( $\mathrm{HCN}$ or $\mathrm{C}_{2} \mathrm{H}$ vs. $\mathrm{HCO}^{+} ; \mathrm{HCN}$ vs. $\mathrm{C}_{2} \mathrm{H}$ ) reveal a high degree of correlation, but sharply non-linear behaviour. In general, all species except $\mathrm{OH}$ increase in abundance more rapidly than linearly with increasing $N\left(\mathrm{HCO}^{+}\right)$at $N\left(\mathrm{HCO}^{+}\right) \gtrsim$ $10^{12} \mathrm{~cm}^{-2}$. Analogous non-linear behaviour in the optical regime is seen in plotting $N(\mathrm{CN})$ vs. $N\left(\mathrm{C}_{2}\right)$ (powerlaw slope around 1.4, see below) or either of these against $N\left(\mathrm{H}_{2}\right)$, but tight linear relationships between species are quite lacking in optical absorption studies.

The manner of taking and analyzing the present observations is discussed in Sect. 2. The main results of this work are discussed in Sect. 3, and a discussion of what is known of the chemistry of simple CN-bearing molecules is presented in Sect. 4.

\section{Observations}

The mm-wave absorption data discussed here were taken at the Plateau de Bure Interferomer during the years 1993-1997. Table 1 shows the list of background sources
Table 1. Background sources and profile rms

\begin{tabular}{lccccc}
\hline Source & $l$ & $b$ & $\sigma_{1 / \mathrm{c}}$ & $\sigma_{1 / \mathrm{c}}$ & $\sigma_{1 / \mathrm{c}}$ \\
& ${ }^{\circ}$ & ${ }^{\circ}$ & $\mathrm{HCN}$ & $\mathrm{HNC}$ & $\mathrm{CN}$ \\
\hline $\mathrm{B} 0212+735$ & 128.93 & 11.96 & 0.040 & 0.066 & 0.108 \\
$\mathrm{~B} 0355+508$ & 150.38 & -1.60 & 0.022 & 0.006 & 0.067 \\
$\mathrm{~B} 0415+379$ & 161.68 & -8.82 & 0.046 & 0.005 & 0.049 \\
$\mathrm{~B} 0528+134$ & 191.37 & -11.01 & 0.016 & 0.023 & 0.024 \\
$\mathrm{~B} 1730-130$ & 12.03 & 10.81 & 0.009 & 0.009 & 0.020 \\
$\mathrm{~B} 2200+420$ & 92.13 & -10.40 & 0.019 & 0.052 & 0.069 \\
$\mathrm{~B} 2251+158$ & 86.11 & -38.18 & 0.008 & 0.014 & \\
\hline
\end{tabular}

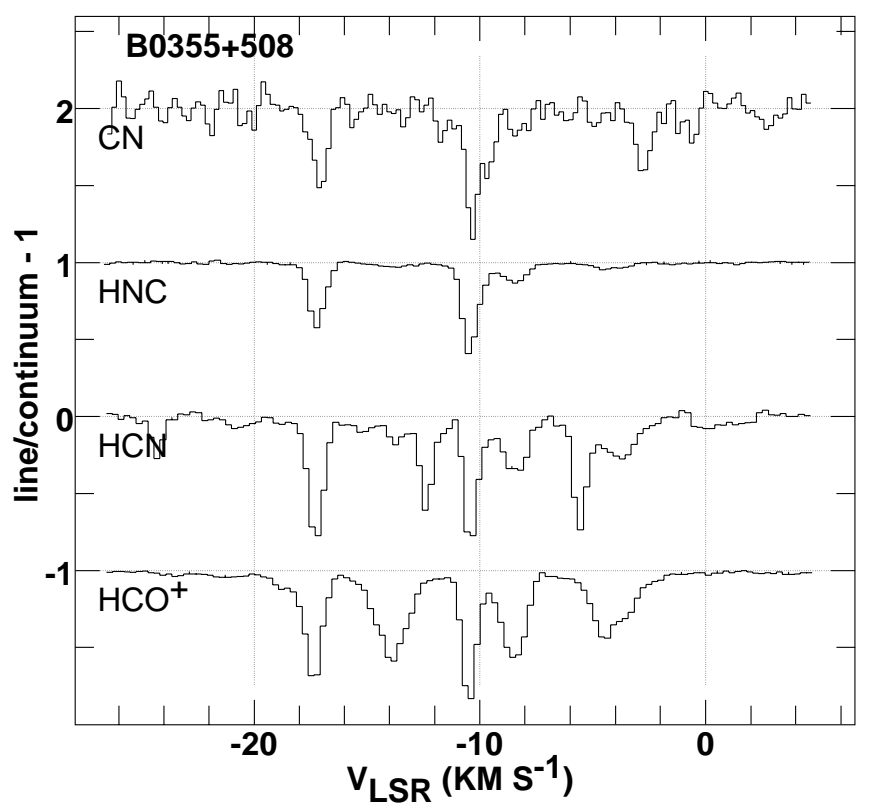

Fig. 2. $\mathrm{HCO}^{+}, \mathrm{CN}, \mathrm{HCN}$ and $\mathrm{HNC}$ absorption spectra toward B0355+508. The HNC spectrum, in which the hyperfine structure only widens and does not create (new) components, most readily shows which of the $\mathrm{HCO}^{+}$features are present in the CN family

observed, along with their galactic coordinates and the rms noise levels achieved in the line/continuum ratio, which is also the rms error in optical depth in the optically thin limit. The mode of observation was identical to that described previously by Lucas \& Liszt (2000) and in our other prior work. All data used for the present survey were taken with a channel separation of $78 \mathrm{kHz}$ and a channel width $(F W H M)$ of $140 \mathrm{kHz}$, corresponding to velocity intervals which are given just below for the various individual species.

We have never succeeded in detecting emission from HCN, the most optically thick of the species discussed here, from the clouds occulting any of our extragalactic continuum sources. Thus we derive all column densities in the limit of no collisional excitation, i.e. $T_{\mathrm{exc}}=T_{\mathrm{cmb}}$. Very weak HCN emission $(0.02 \mathrm{~K})$ was seen some $30^{\prime}$ South of $\zeta$ Oph at the local $\mathrm{HCO}^{+}$emission peak $(0.1 \mathrm{~K}$; 
Liszt 1997) but CN emission is absent at very low levels toward the star itself (Crane et al. 1989; Kopp et al. 1996).

\section{1. $C N$}

We observed the $(1 / 2-1 / 2),(3 / 2-5 / 2)$ and $(1 / 2-3 / 2)$ hyperfine components of the $N=0-1$ absorption line near $113.490 \mathrm{GHz}$, which have LTE line strengths in the proportion 988:3333:1235. As shown in Fig. 1, the lines are split by $3-11 \mathrm{MHz}$ and so are separately resolved. Assuming equilibrium with the cosmic microwave background, the CN column density is related to the integrated optical depth of the $N=0-1$ absorption as $N(\mathrm{CN})=2.2710^{13} \mathrm{~cm}^{-2} \int \tau_{0-1} \mathrm{~d} v$.

Results of Gaussian fitting are given in Table A.1 (see Sect. 2.6). The channel separation and FWHM of the data are $0.206 \mathrm{~km} \mathrm{~s}^{-1}$ and $0.370 \mathrm{~km} \mathrm{~s}^{-1}$, respectively.

\section{2. $\mathrm{HCN}$}

We observed the $F=1-1, F=2-1$ and $F=1-0$ hyperfine components of the $N=0-1$ transition near $88.63 \mathrm{GHz}$, with LTE line strengths in the ratio 1:5:3. As shown in Fig. 1, the lines are split by $1-3 \mathrm{MHz}$ and are separately resolved, though often confused due to blending with other features. Assuming equilibrium with the cosmic microwave background, the HCN column density is related to the optical depth of the $N=0-1$ absorption integrated over all components as $N(\mathrm{HCN})=$ $1.8910^{12} \mathrm{~cm}^{-2} \int \tau_{0-1} \mathrm{~d} v$. Results of Gaussian fitting are given in Table A.2 (see Sect. 2.6). The channel separation and $F W H M$ of the data are $0.264 \mathrm{kms}^{-1}$ and $0.474 \mathrm{~km} \mathrm{~s}^{-1}$, respectively.

\section{3. $H N C$}

We observed the $F=1-1, F=2-1$ and $F=1-0$ hyperfine components of the $N=0-1$ transition near $90.66 \mathrm{GHz}$, with LTE linestrengths in the ratio 1:5:3. As shown in Fig. 1, the hyperfine splittings of the $J=0-1$ absorption of HNC are smaller than typical linewidths, and so are observed as a broadening of the observed components rather than as separate kinematic features. Assuming equilibrium with the cosmic microwave background, the HNC column density is related to the integrated optical depth of the $J=0-1$ absorption as $N(\mathrm{HNC})=1.7610^{12} \mathrm{~cm}^{-2} \int \tau_{0-1} \mathrm{~d} v$. Results of Gaussian fitting are given in Table A.3 (see Sect. 2.6). The channel separation and $F W H M$ of the data are $0.256 \mathrm{~km} \mathrm{~s}^{-1}$ and $0.463 \mathrm{~km} \mathrm{~s}^{-1}$, respectively.

\section{4. $\mathrm{CH}_{3} \mathrm{CN}$}

We observed a band centered at $91.9871 \mathrm{GHz}$, i.e. at the frequency of the strongest hyperfine component of the
$J=5-4$ transition in the $K=0$ ladder, toward $\mathrm{B} 0415+379, \mathrm{~B} 0528+134, \mathrm{~B} 1730-130$, and B2251+158. This band sampled as well the same J-transition in the $K=1$ ladder, whose intensity should be smaller by a factor 0.076 in equilibrium with the cosmic microwave background; yet higher K-ladder transitions falling in the band should have truly negligible population. Although the spectrum is mildly suggestive of a detection toward $3 \mathrm{C} 111$, it is not statistically significant above the $2 \sigma$ level. The spectrum toward 3C 111 had an rms noise in the line/continuum ratio of 0.004 , leading to a 2 sigma upper limit on the integrated optical depth of $\int \tau \mathrm{d} v<0.014 \mathrm{~km} \mathrm{~s}^{-1}$, or $N\left(\mathrm{CH}_{3} \mathrm{CN}\right)<2.410^{11} \mathrm{~cm}^{-2}$, $N\left(\mathrm{CH}_{3} \mathrm{CN}\right) / N\left(\mathrm{HCO}^{+}\right)<0.021$. These are the results quoted in Table 3 and discussed below. $\mathrm{CH}_{3} \mathrm{CN}$ is underabundant compared to the composition of dark clouds like TMC-1 and L134N.

\section{5. $\mathrm{N}_{2} \mathrm{H}^{+}$}

We observed a band around $93.1738 \mathrm{GHz}$, the location of the $J=1-0$ transitions of $\mathrm{N}_{2} \mathrm{H}^{+}$, toward $\mathrm{B} 0355+508$, B0415+379, B0528+134, B1730-130, and B2251+158, but detected no absorption. The best limit is for the gas occulting B0415+379, and this is the datum used in Table 3 and discussed below. The spectrum toward 3C 111 had an rms noise in the line/continuum ratio of 0.0025 , leading to a 2-sigma upper limit on the integrated optical depth of $\int \tau \mathrm{d} v<0.016 \mathrm{~km} \mathrm{~s}^{-1}$, or $N\left(\mathrm{~N}_{2} \mathrm{H}^{+}\right)<2.610^{10} \mathrm{~cm}^{-2}$, $N\left(\mathrm{~N}_{2} \mathrm{H}^{+}\right) / N\left(\mathrm{HCO}^{+}\right)<0.0024$. By comparison, the other lines of sight did not furnish useful data except to confirm that we had not somehow missed large quantities of $\mathrm{N}_{2} \mathrm{H}^{+}$ anywhere. $\mathrm{N}_{2} \mathrm{H}^{+}$is much more strongly underabundant in diffuse gas than $\mathrm{CH}_{3} \mathrm{CN}$.

\subsection{Component fitting}

In Tables A.1-3 of the Appendix we give the results of Gaussian decomposition, done simultaneously to whichever subset of the hyperfine structure was actually observed (all for $\mathrm{HNC}$ and $\mathrm{HCN}$, and part for $\mathrm{CN}$, see Fig. 1). The optical depth quoted at line center pertains to the strongest hyperfine component but was derived from a simultaneous fit to multiple kinematic and hyperfine components, the latter assumed to appear in the LTE ratio, as discussed in Sect. 2.4. The integrated optical depth tabulated is the sum over all hyperfine components for a given kinematic feature, either derived directly (for HCN and $\mathrm{HNC}$ ) or (for $\mathrm{CN}$ ) by a simple scaling to account for that (smaller) fraction of the spectrum which was not actually observed.

We always fit the observed line profile (line/continuum ratio) using saturated gaussians of the form $\exp \left(-\tau_{0} \times\right.$ $\left.\exp \left(-\left(v-v_{0}\right)^{2} / 2 \sigma_{v}{ }^{2}\right)\right)$, simultaneously considering all the accessible hyperfine structure. Thus the optical depths and linewidths which are presented in the tables are consistent 

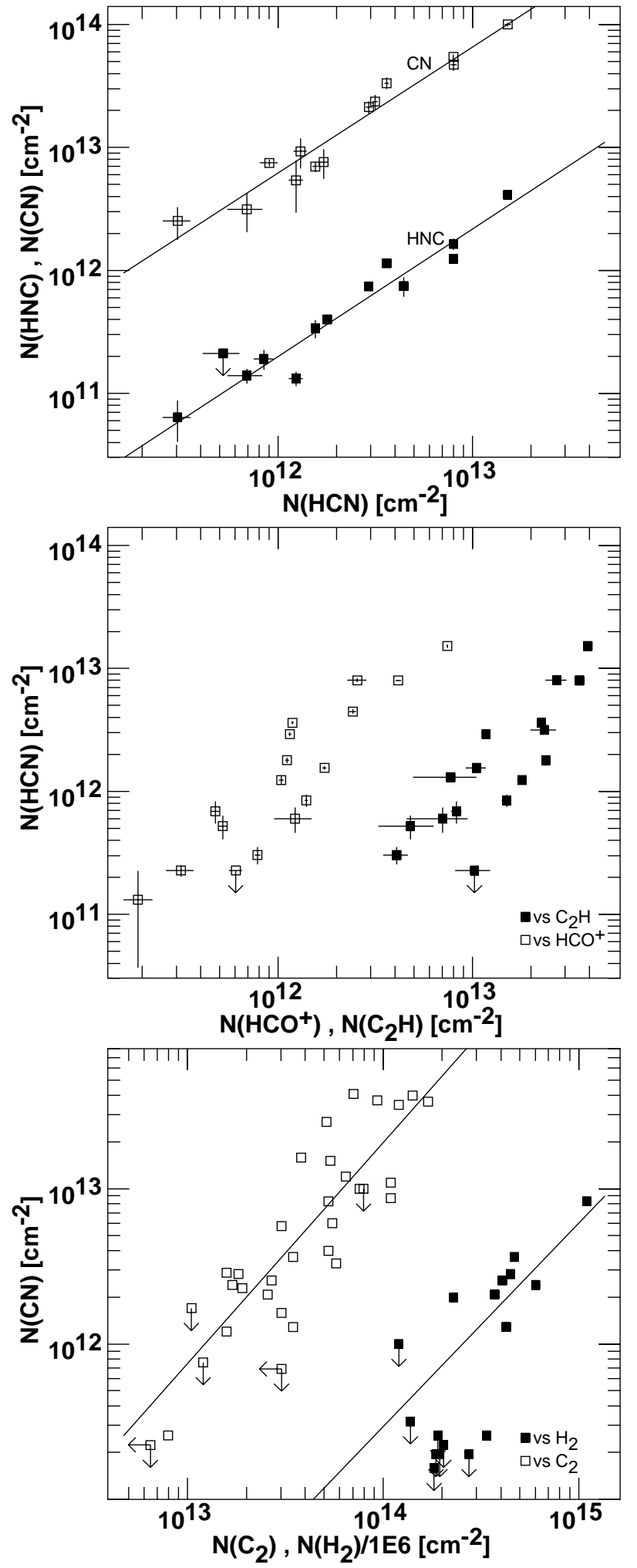

Fig. 3. $\mathrm{HCN}, \mathrm{CN}, \mathrm{HNC}, \mathrm{HCO}^{+}, \mathrm{C}_{2} \mathrm{H}, \mathrm{C}_{2}$ and $\mathrm{H}_{2}$ column densities compared at radio and optical wavelengths. Top: $N(\mathrm{CN})$ (open) and $N(\mathrm{HNC})$ (filled) vs. $N(\mathrm{HCN})$. Middle: $N(\mathrm{HCN})$ vs. $N\left(\mathrm{HCO}^{+}\right)$(open) and $N\left(\mathrm{C}_{2} \mathrm{H}\right)$ (filled). Bottom, $N(\mathrm{CN})$ vs. $N\left(\mathrm{C}_{2}\right)$ and $N\left(\mathrm{H}_{2}\right)$ at optical and $u v$-wavelengths, from the 1995 version of the compilation originally in Federman et al. (1994) with each other and with the degree of saturation which occurs in the data. The fitted linewidths are not artifically increased by line saturation.

As seen in Fig. 2, the intrinsic kinematic structure along a given line of sight can introduce substantial confusion as to which clouds contain which molecule. In each case, we used our $\mathrm{HCO}^{+}$profile as a template and did a simultaneous fit, species-by-species, to all of the hyperfine and kinematic components which were observed. The FWHM linewidths in Tables A1-A3 of the Appendix all include a contribution from the intrinsic broadening in the spectrometer which, as noted above, has channel halfpower widths somewhat wider than the channel separation itself. Where the linewidths are plotted (Fig. 5), this contribution has been subtracted in quadrature from the tabulated value.

\section{Systematics}

\subsection{Abundance and linewidth comparisons within the family of small CN-bearing molecules}

Figure 3 at top shows that $N(\mathrm{CN}), N(\mathrm{HCN})$, and $N$ (HNC) have the kind of tight linear relationship previously demonstrated for $\mathrm{OH}$ and $\mathrm{HCO}^{+}$(Liszt \& Lucas 1996; Lucas \& Liszt 1996). The slopes of both regression lines in Fig. 3 are indistinguishable from unity $(1.03 \pm 0.08$ in either case) with mean ratios (weighted by the variance $)$ of $\langle N(\mathrm{HNC}) / N(\mathrm{HCN})\rangle=0.21 \pm 0.05$ and $\langle N(\mathrm{CN}) / N(\mathrm{HCN})\rangle=6.8 \pm 1.0$.

Figure 5 shows a comparison of the FWHM linewidths derived from profile decomposition (see the tables in the Appendix), corrected for finite resolution. The $\mathrm{HCN}, \mathrm{HNC}$, and $\mathrm{HCO}^{+}$linewidth intercomparisons show no deviation from the condition of equal linewidths, but $\mathrm{CN}$ seems to have a slight tendency to be narrower than $\mathrm{HCN}$ and a more marked tendency to be narrower than $\mathrm{HCO}^{+}$. $\mathrm{CN}$ is typically observed to be narrower than $\mathrm{CH}$ in optical spectra, where the difference is usually interpreted as suggesting a spatial segregration of $\mathrm{CN}$ into the denser core regions of a cloud (Crawford 1995).

In this work, CN was observed with a somewhat better spectral resolution, owing to its higher rest frequency and the fixed nature of the channel widths in the autocorrelator. Although it seems unlikely that resolution dominates the linewidth comparison in Fig. $5-\mathrm{HCN}$ and $\mathrm{HCO}^{+}$are observed at nearly the same frequency and resolution this should certainly be checked. If $\mathrm{CN}$ really is narrower than $\mathrm{HCN}$, this is extremely interesting because the tight correlation of their column densities militates against any really large-scale spatial segregation, such as isolating $\mathrm{CN}$ in a denser core. Instead, there would have to be a mix of species and conditions throughout the gas such that line of sight average column densities vary in fixed proportion, and the CN linewidth would be smaller throughout the cloud, or at least down to scales much finer than we can discern directly. 


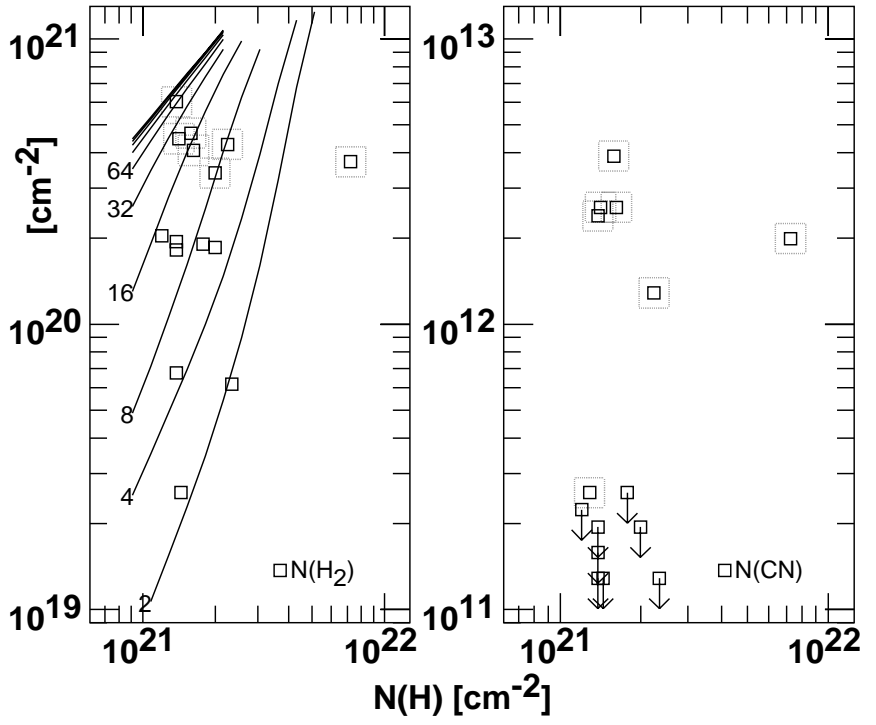

Fig. 4. $N\left(\mathrm{H}_{2}\right)$ (left) and $N(\mathrm{CN})$ plotted against the total $\mathrm{H}-$ column density $N(\mathrm{H})=N(\mathrm{H} \mathrm{I})+2 * N\left(\mathrm{H}_{2}\right)$ for lines of sight with $N(\mathrm{CN})$ observed at optical wavelengths (as in the bottom panel of Fig. 3). For convenience, datapoints corresponding to sightlines with optically-measured CN column densities have been outlined. Superposed in the left-hand panel are theoretical $\mathrm{H}_{2}$-formation curves for uniformly-illuminated gas clots of constant total density $n(\mathrm{H})$ as given $\left(\mathrm{cm}^{-3}\right)$ at their left-hand edges. $N(\mathrm{H})$ and $N\left(\mathrm{H}_{2}\right)$ are from Savage et al. (1977)

\subsection{Abundance comparisons across families of species observed at $\mathrm{mm}$-wavelengths}

Figure 3 in the middle shows the variation of $\mathrm{HCN}$ with respect to $\mathrm{HCO}^{+}$and $\mathrm{C}_{2} \mathrm{H}$. As we have shown before, comparisons across chemical families reveal sharply nonlinear behaviour; although the variations of $\mathrm{HCN}$ with $\mathrm{HCO}^{+}$and $\mathrm{C}_{2} \mathrm{H}$ may appear similar, the $\mathrm{C}_{2} \mathrm{H}-\mathrm{HCO}^{+}$relationship is also non-linear, with the same increase in $N\left(\mathrm{C}_{2} \mathrm{H}\right)$ at $N\left(\mathrm{HCO}^{+}\right) \approx 110^{12} \mathrm{~cm}^{-2}$ (Paper I). $\mathrm{C}_{2} \mathrm{H}$ however has something of a plateau at small $N\left(\mathrm{HCO}^{+}\right)$, where the mean of $N\left(\mathrm{C}_{2} \mathrm{H}\right) / N\left(\mathrm{HCO}^{+}\right)$is high. The $N(\mathrm{HCN}) / N\left(\mathrm{C}_{2} \mathrm{H}\right)$ ratio varies from (roughly) 0.08 to 0.3 , increasing with column density.

Taken as unweighted averages over all observed features, we find $\left\langle N(\mathrm{HCN}) / N\left(\mathrm{HCO}^{+}\right)\right\rangle=1.47 \pm 0.86$, $<N(\mathrm{HCN}) / N\left(\mathrm{C}_{2} \mathrm{H}\right)>=0.155 \pm 0.094$; in Paper I we quoted $<N\left(\mathrm{C}_{2} \mathrm{H}\right) / N\left(\mathrm{HCO}^{+}\right)>=14.5 \pm 6.7$ for the unweighted mean. When the averages are weighted by the fractional errors of the column density, favoring higher column density sources, we have $\left\langle N(\mathrm{HCN}) / N\left(\mathrm{HCO}^{+}\right)\right\rangle$ $=1.97 \pm 0.43$ and $<N(\mathrm{HCN}) / N\left(\mathrm{C}_{2} \mathrm{H}\right)>=0.23 \pm 0.57$.

\subsection{Insights from optical absorption data}

Figure 3 at bottom shows optically-determined $\mathrm{CN}, \mathrm{C}_{2}$, and $\mathrm{H}_{2}$ column densities from the updated version of the summary compilation of Federman et al. (1994).
It is lamentable that there are so few lines of sight for which both molecular hydrogen and $\mathrm{CN}$ have been observed. In any case, although the relationships in Fig. 3 at the bottom are non-linear (power-law slopes of 1.42 and 1.31 reading from left to right) they provide a useful calibration of absolute abundances. For instance, at $N(\mathrm{CN})=2.110^{12} \mathrm{~cm}^{-2}$, the fitted curve gives $N\left(\mathrm{H}_{2}\right)=$ $4.210^{20} \mathrm{~cm}^{-2}$. This can be transferred to the fits at the top of Fig. 3 to infer $N(\mathrm{HCN})=3.110^{11} \mathrm{~cm}^{-2}$ and then to Fig. 3 at the middle for (very) approximately $N\left(\mathrm{HCO}^{+}\right) \approx$ $7.210^{11} \mathrm{~cm}^{-2}$, or $N\left(\mathrm{HCO}^{+}\right) / N\left(\mathrm{H}_{2}\right) \approx 1.710^{-9}$. This is near the single value $N\left(\mathrm{HCO}^{+}\right) / N\left(\mathrm{H}_{2}\right)=210^{-9}$ that we have used for a variety of purposes recently (Liszt \& Lucas 2000), most especially to show that the observed amounts of $\mathrm{CO}$ in diffuse clouds follow directly from recombination of this amount of $\mathrm{HCO}^{+}$with electrons in standard cloud models.

As inferred from this train of thought, the $\mathrm{HCO}^{+}$ abundance is found to be slightly smaller $\left(\approx 1.510^{-9}\right)$ at $N\left(\mathrm{H}_{2}\right)=1.010^{21} \mathrm{~cm}^{-2}$. But from this comparison we gain the new insight that the abrupt increases in the abundances of so many species at $N\left(\mathrm{HCO}^{+}\right) \gtrsim 10^{12} \mathrm{~cm}^{-2}$ are actually occurring at $N\left(\mathrm{H}_{2}\right) \approx 0.3-0.510^{21} \mathrm{~cm}^{-2}$.

\subsection{Linear and non-linear functional dependencies}

It is important to understand which parameters and physical conditions are varying in Fig. 3. For instance, at the top there is a factor 50 variation in $N(\mathrm{CN}), N(\mathrm{HCN})$ and $N(\mathrm{HNC})$, but much of this - perhaps a factor 20-30occurs over a much smaller range in $N\left(\mathrm{HCO}^{+}\right)$: a factor 5-6. Thus the total column density of gas probably changes far less than might otherwise be guessed. At the bottom, $N(\mathrm{CN})$ seems to vary by a factor ten or so at $N\left(\mathrm{H}_{2}\right) \approx 310^{20} \mathrm{~cm}^{-2}$, and less rapidly, but still faster than linearly, at higher $N\left(\mathrm{H}_{2}\right)$.

To tie everything together somewhat more tightly, we show in Fig. 4 the variation of $N(\mathrm{CN})$ and $N\left(\mathrm{H}_{2}\right)$ with total gas column density $N(\mathrm{H})=N(\mathrm{H} \mathrm{I})+2 N\left(\mathrm{H}_{2}\right)$ (Savage et al. 1977) for those sightlines in Fig. 3 where $\mathrm{CN}$ has been measured at optical wavelengths. The point is that, while $N(\mathrm{CN})$ and $N\left(\mathrm{H}_{2}\right)$ are strongly coupled at higher $N\left(\mathrm{H}_{2}\right)$ in Fig. 3, neither $N(\mathrm{CN})$ nor $N\left(\mathrm{H}_{2}\right)$ increases with $N(\mathrm{H})$ or extinction along those same directions. Both $N(\mathrm{CN})$ and $N\left(\mathrm{H}_{2}\right)$ vary substantially over a very small range of $N(\mathrm{H})^{1}$.

The theoretical $\mathrm{H}_{2}$-formation curves superposed in Fig. 4 at left were constructed by sampling along the mean line of sight through a uniformly-illuminated spherical gas

\footnotetext{
${ }^{1}$ In general the effect of extraneous atomic hydrogen is a rightward displacement in Fig. 4, causing the data to appear consistent with $\mathrm{H}_{2}$-formation at too low a local number density. The anomalous point at large $N(\mathrm{H})$ in Fig. 4 for the sightline to $\rho$ Oph A is an extreme example. No other sightline in the Copernicus sample had such a large $N(\mathrm{H})$, for any value of $N\left(\mathrm{H}_{2}\right)$.
} 
parcel of constant total density $n(\mathrm{H})$ (Liszt \& Lucas 2000). They suggest that those sightlines with larger $N\left(\mathrm{H}_{2}\right)-$ and so with larger $N(\mathrm{CN})$ - sample denser and perhaps darker gas with a higher local fraction of molecular hydrogen. This in turn leads to the idea that there is some cascade of effects which accounts for the very strong changes in column density among the trace species, largely driven by the final approach of the molecular hydrogen fraction toward unity, but perhaps having as its cause the change in some other agent.

In the most classical terms, an increase in local number density would drive the molecular hydrogen fraction higher at relatively constant extinction, increasing the rate of formation of ancestor molecular ions like $\mathrm{H}_{2} \mathrm{CN}^{+}$, which would then recombine faster at higher density and (if the thermal pressure is even approximately conserved, as is almost the case in our models) somewhat lower temperature. It helps considerably that both the electron fraction and the abundance of the basic building material $\left(\mathrm{C}^{+}\right)$ are dominated by the $\mathrm{C}^{+} / \mathrm{C}^{0}$ ratio, which remains high in diffuse gas at the moderate density and extinction relevant here. Earlier we showed that even a constant abundance (relative to $\mathrm{H}_{2}$ ) of an ancestor ion could give rise to faster-than-linear increases in the abundance of a product molecule via electron-ion recombination in a gas of constant density, specifically $N(\mathrm{CO})$ varying as $N\left(\mathrm{H}_{2}\right)^{2}$ when arising from gas with constant $N\left(\mathrm{HCO}^{+}\right) / N\left(\mathrm{H}_{2}\right)$ (Liszt \& Lucas 2000).

A related matter of interest is the extent to which the molecules we sample may exist in environments which are still partly atomic. In Fig. 6 we show the variation of the $\mathrm{H}_{2}$-fraction with position inside spherical gas parcels of density $n(\mathrm{H})=16$ and $128 \mathrm{H}-n u c l e i \mathrm{~cm}^{-3}$, for five values of the column density across the central line of sight through the parcel. At the panel to the right in this figure, where the number density is large enough that weak $\mathrm{HCO}^{+}$emission might be seen, even low-extinction gas is mostly molecular: the lowest column density considered here is about that of a standard "Spitzer" HI cloud. But at low density, and when small molecular column densities are sampled, the ambient environment of hydrogen gas is likely to be largely atomic.

\section{Chemistry of simple nitrogen-bearing molecules}

\section{1. $C N, H C N$ and $H N C$}

Shown in Table 2 is a comparison across diffuse and dark gas of the chemical abundances of the hydrocarbons studied in Paper I and species relevant to the present work (NH, CN, HCN, HNC). The tabulation indicates that the abundances of HCN and HNC increase very substantially in moving from diffuse to dark gas while $\mathrm{CN}$ increases in abundance only slightly.

As represented in the last column of Table 2 by the work of Van Dishoeck \& Black (1986), models of moderate-density quiescent gas-phase diffuse cloud
Table 2. Relative abundances $10^{8} \times N() / N\left(\mathrm{H}_{2}\right)$

\begin{tabular}{lcccc}
\hline Species & $\zeta$ Oph & This work & TMC-1 & BD-G \\
\hline $\mathrm{OH}$ & 10 & 7 & 30 & 10 \\
$\mathrm{CO}$ & 480 & & 8000 & 41 \\
$\mathrm{HCO}^{+}$ & 0.2 & 0.2 & 0.8 & 0.009 \\
$\mathrm{C}^{+}$ & 26100 & & & 89100 \\
$\mathrm{C}$ & 700 & & & 720 \\
$\mathrm{C}_{2}$ & 3.3 & & & 3.7 \\
$\mathrm{C}_{3}$ & $<0.012$ & & & $10^{-5}$ \\
$\mathrm{CH}$ & 5.4 & $1-2$ & 2 & 3.9 \\
$\mathrm{CH}^{+}$ & 6.3 & & & 0.006 \\
$\mathrm{C}_{2} \mathrm{H}$ & & $1.3-4$ & 7 & 0.4 \\
$\mathrm{C}_{3} \mathrm{H}$ & & $<0.02$ & 0.05 & \\
$\mathrm{C}_{3} \mathrm{H}_{2}$ & & $0.05-0.15$ & 3 & \\
$\mathrm{C}_{4} \mathrm{H}$ & & & 2.0 & \\
$\mathrm{NH}_{\mathrm{CN}}$ & 0.19 & & & 0.10 \\
$\mathrm{HCN}$ & $(0.079)$ & $0.14-0.5$ & 2 & 0.007 \\
$\mathrm{HNC}$ & $(0.016)$ & $0.03-0.10$ & 2 & \\
$\mathrm{CH}_{3} \mathrm{CN}$ & & $<0.004$ & 0.1 & \\
$\mathrm{~N}_{2} \mathrm{H}^{+}$ & & $<0.0004$ & 0.2 & \\
\hline
\end{tabular}

"This work" represents this work, results from Paper I (for $\mathrm{C}_{n} \mathrm{H}_{m}$-family species), unpublished $\mathrm{CH}$ data and a scaling $N(\mathrm{OH})=35 N\left(\mathrm{HCO}^{+}\right)$.

Results for TMC-1 are from Ohishi et al. (1992).

BD-G is $\zeta$ Oph model G of Van Dishoeck \& Black (1986). For $\zeta$ Oph, $\mathrm{C}^{+}$is from Cardelli et al. (1993), NH is from Crawford \& Williams (1997) and HCN and HNC are normalized to $\mathrm{CN}$.

chemistry provide approximately the correct amounts of $\mathrm{NH}$ and $\mathrm{CN}$, but fail to provide the inferred amount of $\mathrm{HCN}$ (which we derive by scaling $N(\mathrm{CN})$ ) by a factor of 10 . However even this degree of agreement may be illusory. The Van Dishoeck \& Black (1986) results were computed for a very much larger carbon abundance than is now accepted (see the entry under $\zeta$ Oph from Cardelli et al. 1993) and the claim is made (see the discussion of Crawford \& Williams 1997) that ordinary gas phase diffuse cloud chemistry fails by a factor of about 40 to reproduce the observed $N(\mathrm{NH})$ and $N(\mathrm{CN})$. If so, the predicted amounts of most molecules would be much smaller than are indicated in the last column of Table 2.

It seems generally accepted that formation of the $\mathrm{CN}-$ bearing molecules proceeds via the reaction $\mathrm{C}^{+}+\mathrm{NH} \rightarrow$ $\mathrm{CN}^{+}+\mathrm{H}$, followed by a series of hydrogen abstractions beginning with $\mathrm{CN}^{+}+\mathrm{H}_{2} \rightarrow \mathrm{HCN}^{+}+\mathrm{H}$, and terminating (forming one of the observed species $\mathrm{CN}, \mathrm{HCN}, \mathrm{HNC}$ ) after one of many possible electron-ion recombinations such as $\mathrm{H}_{2} \mathrm{CN}^{+}+\mathrm{e} \rightarrow \mathrm{HCN}+\mathrm{H}$ (Van Dishoeck \& Black 1986; Federman et al. 1994; Crawford \& Williams 1997). However, the particulars are very much open to question 


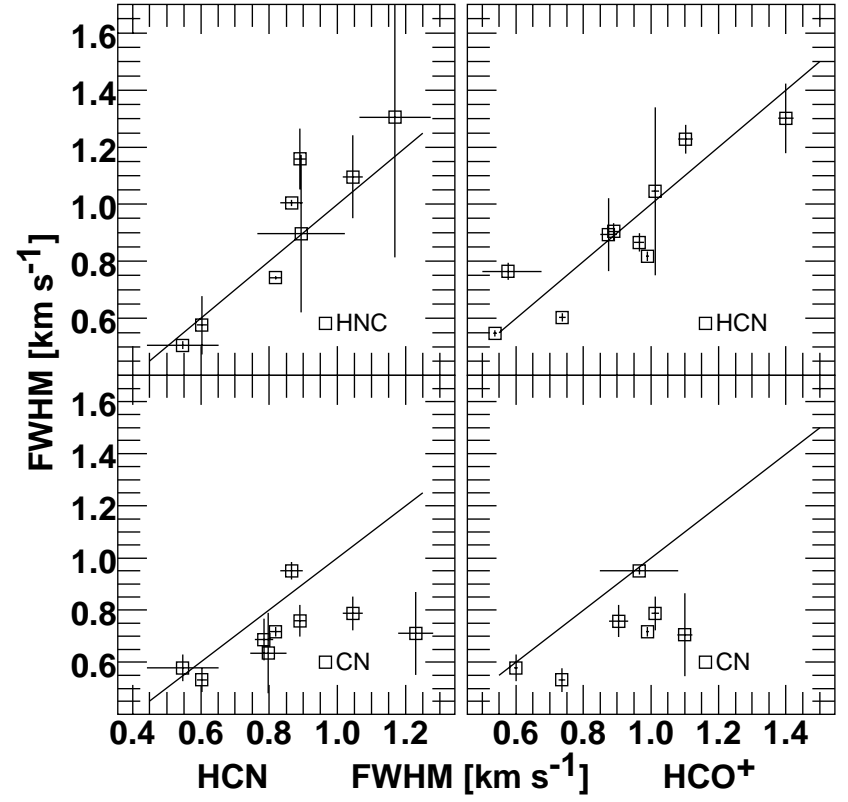

Fig. 5. Comparison of $F W H M$ linewidths. Left: HNC (top) and CN (bottom) vs. HCN. Right: HCN (top) and CN (bottom) vs. $\mathrm{HCO}^{+}$. $\mathrm{HCN}, \mathrm{HNC}$, and $\mathrm{HCO}^{+}$have statistically indistinguishable widths. $\mathrm{CN}$ is noticeably narrower than $\mathrm{HCO}^{+}$

since the needed gas-phase reactions do not proceed nearly fast enough under typical quiescent conditions in diffuse clouds.

Quiescent gas phase chemical models make NH by cosmic ray ionization of $\mathrm{N}$, followed by hydrogen abstraction in the reaction of $\mathrm{N}^{+}$and $\mathrm{H}_{2}$. This and all other gas phase $\mathrm{NH}$ formation reactions are discounted by Crawford \& Williams (1997) in favor of formation on grains; they also argue that the linewidth of $\mathrm{NH}$, which is typical, rules out any formation mechanism involving translationally hot particles in the gas phase, like ions $\left(\mathrm{N}^{+}\right)$accelerated in MHD shocks.

Whether the NH may be formed in gas-phase reactions with fast-moving particles is of interest because a similar formation route is cited as the likely source of the unexplained high abundances of $\mathrm{HCO}^{+}$(i.e. $\mathrm{C}^{+}+$ $\mathrm{OH} \rightarrow \mathrm{CO}^{+}+\mathrm{H}$ ) and $\mathrm{CH}^{+}\left(\right.$via $\mathrm{C}^{+}+\mathrm{H}_{2}$ ) (Falgarone et al. 1995; Hogerheijde et al. 1995; Federman et al. 1996; Joulain et al. 1998). One of these pathways also seems to be needed to explain the observed amounts of $\mathrm{CO}$, as well, and they would help greatly in alleviating the apparent shortfall of the CN-bearing species (through the reaction of $\mathrm{C}^{+}$and $\mathrm{NH}$ ). If such processes do not contribute to alleviating the apparent shortfall of $\mathrm{NH}$, it seems possible that they might not be needed at all to form the CN-bearing species. Alternately, if they do function, they would represent strong sources and sinks of $\mathrm{NH}$ not considered by Crawford \& Williams (1997).

Whether the linewidth of NH rules out an enhanced gas phase formation rate really awaits a calculation of reaction dynamics and subsequent molecular thermalization in the ambient medium (Bucher \& Glinski 1999). For

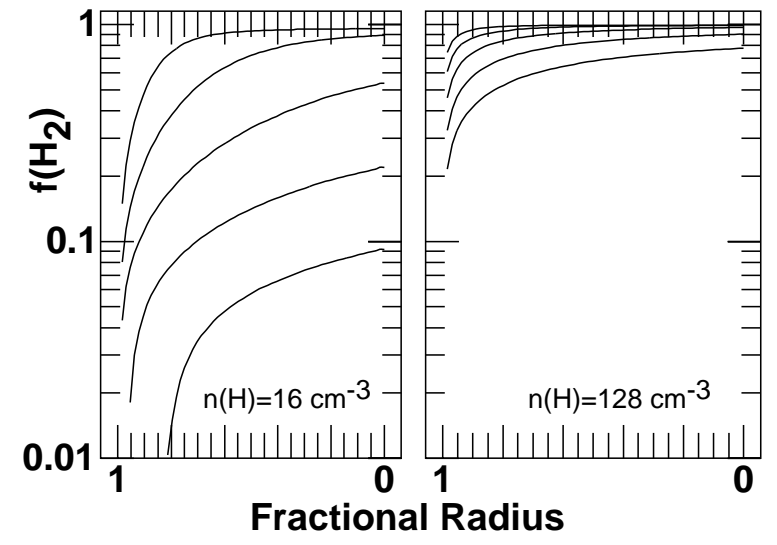

Fig. 6. Theoretical $\mathrm{H}_{2}$-formation curves for uniformlyilluminated spherical gas parcels of constant density $n(\mathrm{H})=$ $n(\mathrm{HI})+2 n\left(\mathrm{H}_{2}\right)=16 \mathrm{~cm}^{-3}$ and $128 \mathrm{~cm}^{-3}$. In each panel the fraction of nuclei in $\mathrm{H}_{2}, f\left(\mathrm{H}_{2}\right)=2 n\left(\mathrm{H}_{2}\right) / n(\mathrm{H})$, is plotted as a function of fractional radius for five values of the total column density along the line of sight intersecting the center of the parcel, $N(\mathrm{H})=0.25,0.5,1,2$ and $410^{21} \mathrm{~cm}^{-2}$

molecules which are formed hot - which includes both those which are formed from translationally hot reactants and those which carry away significant amounts of energy from exoergic chemical reactions - only those which react quickly will disappear before reaching kinetic equilibrium and so exhibit a broader linewidth. $\mathrm{CH}^{+}$, for instance, reacts rapidly with both $\mathrm{H}_{2}$ and electrons, while $\mathrm{NH}$ does not.

\section{2. $\mathrm{CH}_{3} \mathrm{CN}$}

Table 2 shows that $\mathrm{CH}_{3} \mathrm{CN}$ is underabundant in diffuse gas relative to TMC-1, by at least a factor of twenty, or somewhat less, 6, if its abundance relative to $\mathrm{HCO}^{+}$is considered. Given that easily-observed species like $\mathrm{C}_{3} \mathrm{H}_{2}$ and $\mathrm{HCN}$ are underabundant by a similar factor, the limit on $\mathrm{CH}_{3} \mathrm{CN}$ is not that strong and should probably not be considered too surprising. Both of the chemical sequences leading to $\mathrm{CH}_{3} \mathrm{CN}$ in dark gas (Nejad \& Wagenblast 1999), $\left(\mathrm{C}, \mathrm{CH}_{2}, \mathrm{HCN}, \mathrm{H}_{4} \mathrm{C}_{2} \mathrm{~N}^{+}, \mathrm{CH}_{3} \mathrm{CN}\right)$ at early times or $\left(\mathrm{C}_{3} \mathrm{H}\right.$, $\mathrm{C}_{3} \mathrm{~N}, \mathrm{HC}_{3} \mathrm{~N}^{+}, \mathrm{HCN}, \mathrm{H}_{4} \mathrm{C}_{2} \mathrm{~N}^{+}, \mathrm{CH}_{3} \mathrm{CN}$ ) later on, run through $\mathrm{HCN}$.

\section{3. $\mathrm{N}_{2} \mathrm{H}^{+}$}

$\mathrm{N}_{2} \mathrm{H}^{+}$is one of several molecules $\left(\mathrm{N}_{2} \mathrm{H}^{+}, \mathrm{NH}_{3}, \mathrm{SO}_{2}\right)$ which are considered to form only very late in the chemical evolution of dark clouds (Nejad \& Wagenblast 1999). Observationally, $\mathrm{NH}_{3}$ at least is much more widespread than $\mathrm{N}_{2} \mathrm{H}^{+}$and has been seen toward B0355+508 by Nash (1990) and toward 3C 111 in our own unpublished work. However, unpublished VLA results communicated by A. Marscher indicate that reliable $\mathrm{NH}_{3}$ abundances await a 
survey with the upgraded $K$-band receivers at the VLA, and that neither Nash's profiles nor ours (all taken with single dishes), are trustworthy. Unfortunately, $\mathrm{NH}_{3}$ column densities are also unavailable from Marscher's VLA profiles, because only the $(1,1)$ lines were observed.

In molecular clouds, $\mathrm{N}_{2} \mathrm{H}^{+}$forms in appreciable quantities at late times via $\mathrm{H}_{3}{ }^{+}+\mathrm{N}_{2} \rightarrow \mathrm{N}_{2} \mathrm{H}^{+}+\mathrm{H}_{2}$, i.e. when the ionization fraction has dropped and when $\mathrm{CO}$ has been depleted from the gas phase, encouraging a higher abundance of $\mathrm{H}_{3}{ }^{+}$. It is known for being detectable only in darker regions.

Toward 3C 111, $N\left(\mathrm{~N}_{2} \mathrm{H}^{+}\right) / N\left(\mathrm{HCO}^{+}\right)<0.002(2-$ sigma) as compared to 0.25 in TMC-1. This is by far the best limit we have ever been able to set on an undetected species. 3C 111 is actually seen through a small hole (region of lower than average extinction) in an outlying cloud in the Taurus cloud complex; a discussion of the viewing geometry is given by Lucas \& Liszt (1998).

\section{Summary}

We showed that the abundances of $\mathrm{CN}, \mathrm{HCN}$ and $\mathrm{HNC}$ vary in fixed proportion 6.8:1:0.21 respectively. This is rather different from conditions in TMC-1, where their abundances are in the ratio 6.8:4.5:4.5. The fractional abundances of HCN and HNC increase substantially between diffuse and dark gas but that of $\mathrm{CN}$ increases only by factor of a few. Nonetheless, HCN and HNC are much more abundant in diffuse gas than predicted by models of quiescent low-density gas-phase chemistry, with an intrinsic variation over the approximate range $N(\mathrm{HCN}) / N\left(\mathrm{H}_{2}\right) \approx 1.4-5.010^{-9}$ for $N\left(\mathrm{HCO}^{+}\right) / N\left(\mathrm{H}_{2}\right)=$ $2.010^{-9}$. The latter value is confirmed by comparing our measurements of $\mathrm{CN}, \mathrm{HCN}$, and $\mathrm{HCO}^{+}$with the lamentably slight body of optical absorption data for lines of sight where both $\mathrm{CN}$ and $\mathrm{H}_{2}$ have been observed.

The linewidths of $\mathrm{HCN}, \mathrm{HNC}$, and $\mathrm{HCO}^{+}$are sensibly identical, but $\mathrm{CN}$ shows a slight tendency to be narrower than HCN and a more pronounced tendency to be narrower than $\mathrm{HCO}^{+}$. If the $\mathrm{CN}$ and $\mathrm{HCN}$ are strongly coupled in column density but different kinematically, this argues against a spatial segregation of $\mathrm{CN}$ into denser regions of a cloud (core), such as is usually invoked to explain the narrower width of $\mathrm{CN}$ absorption lines seen in the optical. Instead, clouds would have to be a mixture of kinematically (and chemically) distinct regions all occurring many times along any one line of sight.

In comparing the abundances of species in the $\mathrm{CN}$ family with the hydrocarbons discussed in Paper I (principally $\mathrm{C}_{2} \mathrm{H}$ and $\mathrm{C}_{3} \mathrm{H}_{2}$ ) or with $\mathrm{HCO}^{+}$, we find strong correlations but sharply non-linear variation. Both the $\mathrm{CN}$-species and hydrocarbons show a strong increase in column density around $N\left(\mathrm{HCO}^{+}\right)=10^{12} \mathrm{~cm}^{-2}$ and all comparisons across families show relative abundance changes by factors of 3 or more. We note that $\mathrm{C}_{2} \mathrm{H}$ and $\mathrm{C}_{3} \mathrm{H}_{2}$ vary in fixed proportion with each other, though not as tightly as for $\mathrm{HCO}^{+}$and $\mathrm{OH}$, or as seen here.
For those sightlines where the column densities of CN, $\mathrm{HI}$, and $\mathrm{H}_{2}$ have been measured optically, it appears that $N(\mathrm{CN})$ turns on abruptly at $N\left(\mathrm{H}_{2}\right) \approx 310^{20} \mathrm{~cm}^{-2}$ and increases faster than linearly at larger $N\left(\mathrm{H}_{2}\right)$, in a regime where the abundance of $\mathrm{H}_{2}$ itself varies widely over a very small range in $N(\mathrm{H})$ (or extinction). Models of $\mathrm{H}_{2}$ formation suggest that the hydrogen is still largely atomic in the gas sampled on sightlines with the smallest column densities of the trace species like CN.

Earlier (Liszt \& Lucas 2000), we showed that $N(\mathrm{CO}) \propto N\left(\mathrm{H}_{2}\right)^{2}$ (approximately) when CO forms in a gas of constant density via recombination of $\mathrm{HCO}^{+}$, and the latter is held in fixed proportion $\left(210^{-9}\right)$ to $\mathrm{H}_{2}$. It follows that much stronger variations in trace abundances would occur if the higher molecular fractions along some sightlines - reflected in higher $N\left(\mathrm{H}_{2}\right)$ and $N\left(\mathrm{HCO}^{+}\right)$arise because the density is higher locally; the sequence of molecular abundances revealed in our work must at least partly reflect the tendency for higher-density regions to harbor higher $\mathrm{H}_{2}$-fractions at a given total hydrogen column density. Of course this cannot be the whole story because the high abundances of the parent ions needed to reproduce our observations of their descendants cannot presently be explained by any agreed-upon physical scenario. Equally important is to understand why the abundances of some species relative to $\mathrm{H}_{2}$ vary so strongly (e.g. $\mathrm{C}_{2} \mathrm{H}, \mathrm{C}_{3} \mathrm{H}_{2}, \mathrm{CN}, \mathrm{HCN}$, and $\mathrm{HCN}$ ) while those of $\mathrm{CH}, \mathrm{OH}$, and $\mathrm{HCO}^{+}$seem nearly fixed.

We failed to find $\mathrm{CH}_{3} \mathrm{CN}$ and $\mathrm{N}_{2} \mathrm{H}^{+}$, meaning that they are at least 10 and 100 times less abundant (relative to $\mathrm{HCO}^{+}$) in diffuse clouds than in TMC-1. The limit on $\mathrm{CH}_{3} \mathrm{CN}$ may not be very significant, given that $\mathrm{C}_{3} \mathrm{H}_{2}$ and $\mathrm{HCN}$ are underabundant by about the same amount but are readily seen along many lines of sight and that, in dark gas, the formation paths to $\mathrm{CH}_{3} \mathrm{CN}$ pass through $\mathrm{HCN}$ at both early and late times. The limit for $\mathrm{N}_{2} \mathrm{H}^{+}$may be more interesting but this species is notoriously hard to detect even in translucent clouds of much higher density than we study.

We discussed what is known of the chemistry of the $\mathrm{CN}-\mathrm{HCN}-\mathrm{HCN}$ species. Although there is a consensus that they form through some chemical pathway involving $\mathrm{NH}$, most likely interacting with $\mathrm{C}^{+}$, the origin of $\mathrm{NH}$ and the mode of interaction are entirely uncertain.

The next papers in this series will discuss the sulfurbearing species $\mathrm{CS}, \mathrm{SO}, \mathrm{SO}_{2}, \mathrm{H}_{2} \mathrm{~S}$ and $\mathrm{HCS}^{+}$, and probably $\mathrm{NH}_{3}$ and $\mathrm{H}_{2} \mathrm{CO}$.

Acknowledgements. We thank Dr. John Black for a close reading of the manuscript.

The National Radio Astronomy Observatory is operated by AUI, Inc. under a cooperative agreement with the US National Science Foundation. IRAM is operated by CNRS (France), the MPG (Germany) and the IGN (Spain). We owe the staff at IRAM (Grenoble) and the Plateau de Bure our thanks for their assistance in taking the data. 


\section{Appendix A: Products of Gaussian fitting}

Table A.1. CN Absorption line decomposition products

\begin{tabular}{lcccc}
\hline Source & $\begin{array}{c}\text { v } \\
\mathrm{km} \mathrm{s}^{-1}\end{array}$ & & $\begin{array}{c}F W H M \\
\mathrm{~km} \mathrm{~s}^{-1}\end{array}$ & $\begin{array}{c}\int \tau \mathrm{d} v \\
\mathrm{~km} \mathrm{~s}^{-1}\end{array}$ \\
\hline B0212+735 & $3.612(0.033)$ & $1.536(0.104)$ & $0.759(0.061)$ & $2.067(0.217)$ \\
B0355+508 & $-17.076(0.024)$ & $0.995(0.073)$ & $0.532(0.045)$ & $0.939(0.106)$ \\
& $-11.197(0.380)$ & $0.115(0.031)$ & $1.621(0.696)$ & $0.331(0.172)$ \\
& $-10.295(0.023)$ & $1.432(0.096)$ & $0.579(0.051)$ & $1.472(0.163)$ \\
& $-8.292(0.073)$ & $0.267(0.041)$ & $0.706(0.159)$ & $0.335(0.092)$ \\
B0415+379 & $-2.458(0.017)$ & $1.431(0.030)$ & $0.951(0.034)$ & $2.415(0.100)$ \\
& $-0.872(0.011)$ & $3.459(0.060)$ & $0.717(0.019)$ & $4.399(0.140)$ \\
B0528+134 & $0.246(0.063)$ & $0.169(0.027)$ & $0.459(0.142)$ & $0.138(0.048)$ \\
B1730-130 & $4.582(0.030)$ & $0.220(0.010)$ & $0.788(0.065)$ & $0.308(0.029)$ \\
$2200+420$ & $-0.974(0.038)$ & $0.852(0.056)$ & $0.687(0.080)$ & $1.038(0.139)$ \\
& $0.019(0.069)$ & $0.367(0.046)$ & $0.635(0.154)$ & $0.413(0.113)$ \\
\hline
\end{tabular}

Table A.2. HCN Absorption line decomposition products

\begin{tabular}{|c|c|c|c|c|}
\hline Source & $\begin{array}{c}v \\
\mathrm{~km} \mathrm{~s}^{-1} \\
\end{array}$ & $\tau_{0}$ & $\begin{array}{c}F W H M \\
\mathrm{~km} \mathrm{~s}^{-1}\end{array}$ & $\begin{array}{c}\int \tau \mathrm{d} v \\
\mathrm{~km} \mathrm{~s}^{-1}\end{array}$ \\
\hline \multirow[t]{2}{*}{ B0212+735 } & $-0.092(0.044)$ & $0.234(0.031)$ & $0.578(0.099)$ & $0.259(0.056)$ \\
\hline & $3.481(0.011)$ & $2.584(0.037)$ & $0.890(0.018)$ & $4.406(0.107)$ \\
\hline \multirow[t]{3}{*}{ B0212+735 } & $-0.104(0.047)$ & $0.238(0.031)$ & $0.605(0.104)$ & $0.276(0.060)$ \\
\hline & $2.655(0.101)$ & $0.204(0.032)$ & $0.811(0.143)$ & $0.318(0.075)$ \\
\hline & $3.517(0.019)$ & $2.750(0.044)$ & $0.805(0.026)$ & $4.241(0.154)$ \\
\hline \multirow[t]{5}{*}{ B0355+508 } & $-17.205(0.009)$ & $1.345(0.036)$ & $0.603(0.015)$ & $1.555(0.057)$ \\
\hline & $-13.770(0.049)$ & $0.191(0.010)$ & $1.301(0.123)$ & $0.477(0.051)$ \\
\hline & $-10.406(0.006)$ & $1.828(0.032)$ & $0.547(0.010)$ & $1.915(0.049)$ \\
\hline & $-8.397(0.027)$ & $0.386(0.011)$ & $1.228(0.051)$ & $0.907(0.046)$ \\
\hline & $-4.632(0.073)$ & $0.163(0.007)$ & $2.104(0.154)$ & $0.655(0.056)$ \\
\hline \multirow[t]{3}{*}{ B0415+379 } & $-3.975(0.274)$ & $0.107(0.016)$ & $1.867(0.486)$ & $0.382(0.115)$ \\
\hline & $-2.518(0.016)$ & $2.540(0.045)$ & $0.866(0.032)$ & $4.216(0.174)$ \\
\hline & $-0.926(0.011)$ & $5.442(0.074)$ & $0.818(0.017)$ & $8.532(0.212)$ \\
\hline B0528+134 & $9.609(0.014)$ & $0.411(0.007)$ & $1.045(0.029)$ & $0.824(0.027)$ \\
\hline \multirow[t]{2}{*}{ B1730-130 } & $4.914(0.070)$ & $0.094(0.006)$ & $0.894(0.128)$ & $0.161(0.026)$ \\
\hline & $5.983(0.199)$ & $0.034(0.005)$ & $0.986(0.382)$ & $0.065(0.027)$ \\
\hline \multirow[t]{2}{*}{ B2200+420 } & $-1.426(0.018)$ & $1.111(0.015)$ & $0.785(0.027)$ & $1.670(0.063)$ \\
\hline & $-0.527(0.035)$ & $0.451(0.014)$ & $0.799(0.053)$ & $0.690(0.050)$ \\
\hline B2251+158 & $-9.545(0.048)$ & $0.054(0.003)$ & $1.169(0.105)$ & $0.120(0.013)$ \\
\hline \multirow[t]{6}{*}{ B0355+508 } & $-17.207(0.007)$ & $1.390(0.017)$ & $0.583(0.012)$ & $1.553(0.036)$ \\
\hline & $-13.727(0.050)$ & $0.164(0.007)$ & $1.419(0.125)$ & $0.445(0.044)$ \\
\hline & $-10.407(0.005)$ & $1.851(0.026)$ & $0.542(0.009)$ & $1.923(0.041)$ \\
\hline & $-8.433(0.025)$ & $0.367(0.009)$ & $1.335(0.048)$ & $0.940(0.040)$ \\
\hline & $-4.633(0.066)$ & $0.154(0.006)$ & $2.156(0.139)$ & $0.637(0.048)$ \\
\hline & $-2.134(0.000)$ & $0.095(0.000)$ & $1.441(0.000)$ & $0.262(0.000)$ \\
\hline
\end{tabular}


Table A.3. HNC Absorption line decomposition products

\begin{tabular}{lcccc}
\hline Source & $\begin{array}{c}v \\
\mathrm{~km} \mathrm{~s}^{-1}\end{array}$ & $\begin{array}{c}F W H M \\
\mathrm{~km} \mathrm{~s}^{-1}\end{array}$ & $\begin{array}{c}\int \tau \mathrm{d} v \\
\mathrm{~km} \mathrm{~s}^{-1}\end{array}$ \\
\hline B0212+735 & $-0.512(0.035)$ & $0.341(0.069)$ & $0.226(0.048)$ & $0.148(0.044)$ \\
& $3.766(0.042)$ & $0.455(0.024)$ & $1.159(0.107)$ & $1.009(0.107)$ \\
B0355+508 & $-17.172(0.004)$ & $0.383(0.006)$ & $0.577(0.014)$ & $0.423(0.012)$ \\
& $-13.713(0.104)$ & $0.016(0.001)$ & $1.910(0.270)$ & $0.058(0.009)$ \\
& $-11.229(0.150)$ & $0.020(0.003)$ & $0.735(0.355)$ & $0.029(0.015)$ \\
& $-10.384(0.005)$ & $0.676(0.009)$ & $0.498(0.014)$ & $0.645(0.020)$ \\
& $-8.564(0.022)$ & $0.074(0.001)$ & $1.577(0.060)$ & $0.223(0.009)$ \\
B0415+379 & $-4.241(0.063)$ & $0.024(0.001)$ & $1.715(0.156)$ & $0.080(0.008)$ \\
& $-2.467(0.004)$ & $0.367(0.002)$ & $1.004(0.011)$ & $0.706(0.009)$ \\
B0528+134 & $-0.874(0.002)$ & $1.662(0.006)$ & $0.743(0.006)$ & $2.365(0.021)$ \\
B1730-130 & $9.382(0.052)$ & $0.105(0.008)$ & $0.964(0.146)$ & $0.193(0.033)$ \\
& $4.890(0.093)$ & $0.022(0.003)$ & $0.897(0.275)$ & $0.038(0.013)$ \\
B2200+420 & $-1.733(0.305)$ & $0.007(0.003)$ & $0.897(0.918)$ & $0.011(0.013)$ \\
B2251+158 & $-10.092(0.190)$ & $0.018(0.004)$ & $1.305(0.492)$ & $0.046(0.019)$ \\
\hline
\end{tabular}

\section{References}

Adams, W. S. 1941, ApJ, 93, 11

Black, J. H., \& van Dishoeck, E. F. 1991, ApJ, 369, L9

Bucher, M. E., \& Glinski, R. J. 1999, MNRAS, 308, 29

Cardelli, J. A., Mathis, J. S., Ebbets, D. C., \& Savage, B. D. 1993, ApJ, 402, L17

Crane, P., Hegyi, D. J., Kutner, M. L., \& Mandolesi, N. 1989, ApJ, 346, 136

Crawford, I. A. 1995, MNRAS, 277, 458

Crawford, I. A., \& Williams, D. A. 1997, MNRAS, 291, L53

Falgarone, E., Pineau des Forêts, G., \& Roueff, E. 1995, A\&A, 300,870

Federman, S. R., Rawlings, J. M. C., Taylor, S. D., \& Williams, D. A. 1996, MNRAS, 279, L41

Federman, S. R., Strom, C. J., Lambert, D. L., et al. 1994, ApJ, 424, 772

Hogerheijde, M. R., De Geus, E. J., Spaans, M., Van Langevelde, H. J., \& Van Dishoeck, E. F. 1995, ApJ, 441, L93

Joulain, K., Falgarone, E., Pineau des Forêts, G., \& Flower, D. 1998, A\&A, 340, 241

Kopp, M., Gerin, M., Roueff, E., \& Le Bourlot, J. 1996, A\&A, 305,558
Liszt, H. S. 1997, A\&A, 322, 962

Liszt, H. S., \& Lucas, R. 1994, ApJ, 431, L131

Liszt, H. S., \& Lucas, R. 1996, A\&A, 314, 917

Liszt, H. S., \& Lucas, R. 2000, A\&A, 355, 333

Lucas, R., \& Liszt, H. 1998, A\&A, 337, 246

Lucas, R., \& Liszt, H. S. 1993, A\&A, 276, L33

Lucas, R., \& Liszt, H. S. 1994, A\&A, 282, L5

Lucas, R., \& Liszt, H. S. 1996, A\&A, 307, 237

Lucas, R., \& Liszt, H. S. 2000, A\&A, 355, 327

McKellar, A. 1940, PASP, 52, 187

Nash, A. G. 1990, A\&AS, 72, 303

Nejad, L. A. M., \& Wagenblast, R. 1999, A\&A, 350, 204

Ohishi, M., Irvine, W., \& Kaifu, N. 1992, in Astrochemistry of cosmic phenomena: Proceedings of the 150th Symposium of the International Astronomical Union, held at Campos do Jordao, Sao Paulo, Brazil, August 5-9, 1991, ed. P. D. Singh (Dordrecht: Kluwer)

Savage, B. D., Drake, J. F., Budich, W., \& Bohlin, R. C. 1977, ApJ, 216, 291

Swings, P., \& Rosenfeld, L. 1937, ApJ, 86, 483

Thaddeus, P. 1972, ARA\&A, 10, 305

Van Dishoeck, E. F., \& Black, J. H. 1986, ApJS, 62, 109 